\title{
The role of advanced end-use technologies in long-term climate change mitigation: the interlinkage between primary bioenergy and energy end-use
}

\author{
Junichi Tsutsui ${ }^{1}$ (D) $\cdot$ Hiromi Yamamoto ${ }^{2} \cdot$ Shogo Sakamoto $^{1} \cdot$ Masahiro Sugiyama $^{3}$
}

Received: 1 May 2018 / Accepted: 17 August 2020/Published online: 27 August 2020

(C) The Author(s) 2020, corrected publication 2020

\begin{abstract}
The role of advanced end-use technologies has been investigated in multiple series of scenarios using an integrated assessment model BET-GLUE, which comprises an energyeconomic module (BET) and a bioenergy-land-use module (GLUE). The scenarios consider different technology assumptions on the availability of bioenergy with carbon capture and storage (BECCS) and end-use efficiencies featuring electrification under a wide range of carbon price trajectories, which start at $1-690 \mathrm{USD} / \mathrm{tCO}_{2}$ in 2030 , increase at $4.5 \%$ year, and level off in 2100 . This scenario design allows close examination of energy, economic, and environmental implications of different levels of policy stringency and carbon budgets. While improving end-use efficiencies consistently decrease policy costs for a wide range of carbon budgets, the value of BECCS availability in terms of cost reduction is crucial only in a limited range toward lower budgets. Constraints on BECCS, including those indirectly imposed by the limited bioenergy supply, also tend to narrow the lower range of attainable budget levels, indicating technological and economic challenges, although they may have an impact on reducing the total budget including land-use emissions. Overall, the advanced end-use efficiency has a significant effect on the required level of policy stringency for a given climate goal, so that it can compensate for the biomass constraints.
\end{abstract}

This article is part of the Special Issue on "Assessing Large-scale Global Bioenergy Deployment for Managing Climate Change (EMF-33)" edited by Steven Rose, John Weyant, Nico Bauer, Shinichiro Fuminori, Petr Havlik, Alexander Popp, Detlef van Vuuren, and Marshall Wise.

Electronic supplementary material The online version of this article (https://doi.org/10.1007/s10584-02002839-7) contains supplementary material, which is available to authorized users.

Junichi Tsutsui

tsutsui@criepi.denken.or.jp

1 Environmental Science Research Laboratory, Central Research Institute of Electric Power Industry, Abiko, Japan

2 Energy Innovation Center, Central Research Institute of Electric Power Industry, Yokosuka, Japan

3 Institute for Future Initiatives, University of Tokyo, Tokyo, Japan 
Keywords BET-GLUE - Bioenergy with carbon capture and storage $\cdot$ Advanced end-use technologies

\section{Introduction}

The present study investigates the role of advanced end-use technologies (AETs) in long-term climate mitigation by using an integrated assessment model (IAM), named BET (Yamamoto et al. 2014), as one of the specific studies conducted in the Stanford Energy Modeling Forum's 33rd model comparison exercise (EMF-33, Bauer et al. 2018). The EMF-33 deals with various issues of large-scale global bioenergy deployment for achieving climate policy objectives, including assumptions on biomass feedstock supply and bioenergy technologies availability and interactions with land-use and food production sectors. BET is one of the 11 participant models and categorized into a multi-regional, inter-temporal general equilibrium model with a detailed energy system and an aggregated representation of the macroeconomy that follows the approach of optimal economic growth. Differently from other types of IAMs, this model explicitly handles energy service demand and end-use technologies in a global macroeconomic framework. This structure allows a systematic approach to examine trade-offs between advanced bioenergy technologies (ABTs) and end-use efficiencies from long-term scenarios across different dimensions of mitigation policies and technologies. The AET is defined here as modern end-use equipment that leads to a significant increase in energy efficiency.

It has been widely recognized that efficiency and relevant electrification have a key role in reducing the use of energy and fostering large-scale decarbonization both in the short and in the long term (Clarke et al. 2014). This is confirmed by recent trends, which include a growing end-use electrification expansion and energy efficiency improvements (IEA 2017, 2018). These transitions have been explored by IAMs from a broad perspective (e.g., Kriegler et al. 2014; Krey et al. 2014; Sugiyama et al. 2014; Yamamoto et al. 2014), as well as focusing on specific sectors (e.g., Kermeli et al. 2014; Guneralp et al. 2017; Edelenbosch et al. 2017; Mittal et al. 2017). More recently, a possibility of unprecedented energy efficiency improvements has been presented in achieving very ambitious climate policy goals (Grubler et al. 2018).

While energy efficiency improvements by advanced technologies are one of the most fundamental strategies for climate change mitigation, ABTs that produce low-carbon energy and enable negative net emissions (van Vuuren et al. 2013; Edmonds et al. 2013; Smith et al. 2016), such as bioenergy with carbon capture and storage (BECCS), have also been getting more attention for their crucial role in attaining ambitious mitigation goals (Clarke et al. 2014). Typically, past studies have dealt with these two strategies separately using an aggregate indicator; on the one hand, improvements in AETs have been analyzed in a way varying the overall improvements of final energy intensity, whereas on the other hand ABTs have been varied as part of a broader sensitivity regarding carbon capture and storage (CCS). However, the two strategies are of the nature that should complement each other, and it is necessary to clarify what end-use energy efficiency improvements could contribute to energy sector emissions reductions in combination with large-scale bioenergy deployment.

The present work is based on a previous study of ours (Yamamoto et al. 2014) that addressed the role of end-use technologies and found that electrification with AETs is a robust strategy for achieving stringent climate policy objectives with less economic impact. We will build off this work by evaluating the interlinkage between primary bioenergy and energy end- 
use within the EMF-33 framework. Our study is motivated by the need to understand general interlinkage between different technologies for expanding low-carbon energy supply and associated demand-side electrification while reducing the overall energy demand. While the current scope takes an important step forward, it is not exhaustive and future work should explore other critical aspects, such as variable renewables and electricity storage in the context of modern demand-side flexibility.

To investigate key issues relevant to large-scale bioenergy deployment, the EMF-33 has designed a variety of scenario experiments into two phases: biomass supply and bioenergy demand. We use the latter demand scenarios, consisting of the combination of different levels of policy stringency for climate mitigation and different constraints on ABTs. Besides these, we have built a number of additional scenarios including the AET dimension over a wide range of policy stringency levels. Sections 2 and 3 describe the model and scenario experiments, Section 4 presents and discusses the results, and finally, Section 5 provides concluding remarks.

\section{Methodology}

BET includes energy supply, energy end-use, and macroeconomic modules. The model divides the world into 13 regions and solves the economic equilibrium problem by applying inter-temporal optimization methods by 10-year steps on the domain 2010-2230. Here, results are shown at the global level over the period 2010-2100. The model basically follows the MARKAL-MACRO formulation (Loulou et al. 2004) for the macroeconomic production function and the structure of the energy supply module from primary to final energy. The end-use module represents end-use technologies, that is, the conversion from final energy to energy services. Service demands are used as energy inputs to the production function and then endogenously determined. Table 1 shows the categories of energy services and the corresponding end-use technologies, which are substitutable within the same service category. The energy supply module includes mining of fossil fuels, fuel conversion, and power generation. The details including basic scenarios and model parameters, such as costs and efficiencies in the energy system, are described in the Electronic Supplemental Material (ESM) (sections 1-3, Figs. ESM.1-ESM.5 and Tables ESM.1-ESM.12). The components of non$\mathrm{CO}_{2}$ greenhouse gases and the interaction between energy system and climate are not considered in the present study.

In the energy supply module, biomass feedstock potentials are retrieved from a separate model, called GLUE (a multi-regional global-land-use-and-energy model, Yamamoto et al. 2001), which solves the system of land-use and biomass flow balance by recursive dynamics under a set of conditions including food and wood demand. GLUE is also employed to quantify land-use $\mathrm{CO}_{2}$ emissions through the calculation of the changes in carbon stocks in soil, above- and below-ground biomass, and woods for industrial use.

GLUE provides potential supplies for 18 types of primary biomass, and BET aggregates them through an exogenous 5-step supply function into four categories: (1) energy crops, such as switchgrass in temperate latitudes and eucalyptus in the tropics (only second-generation crops are considered, excluding first generation), (2) fuelwood from forestry whole logs, (3) wet residues from food biomass including animal dung and human wastes, and (4) residues from food biomass in dry form and woody biomass discharged from industrial material recycling. The secondary energy converted from these feedstocks can be of four types: (1) 


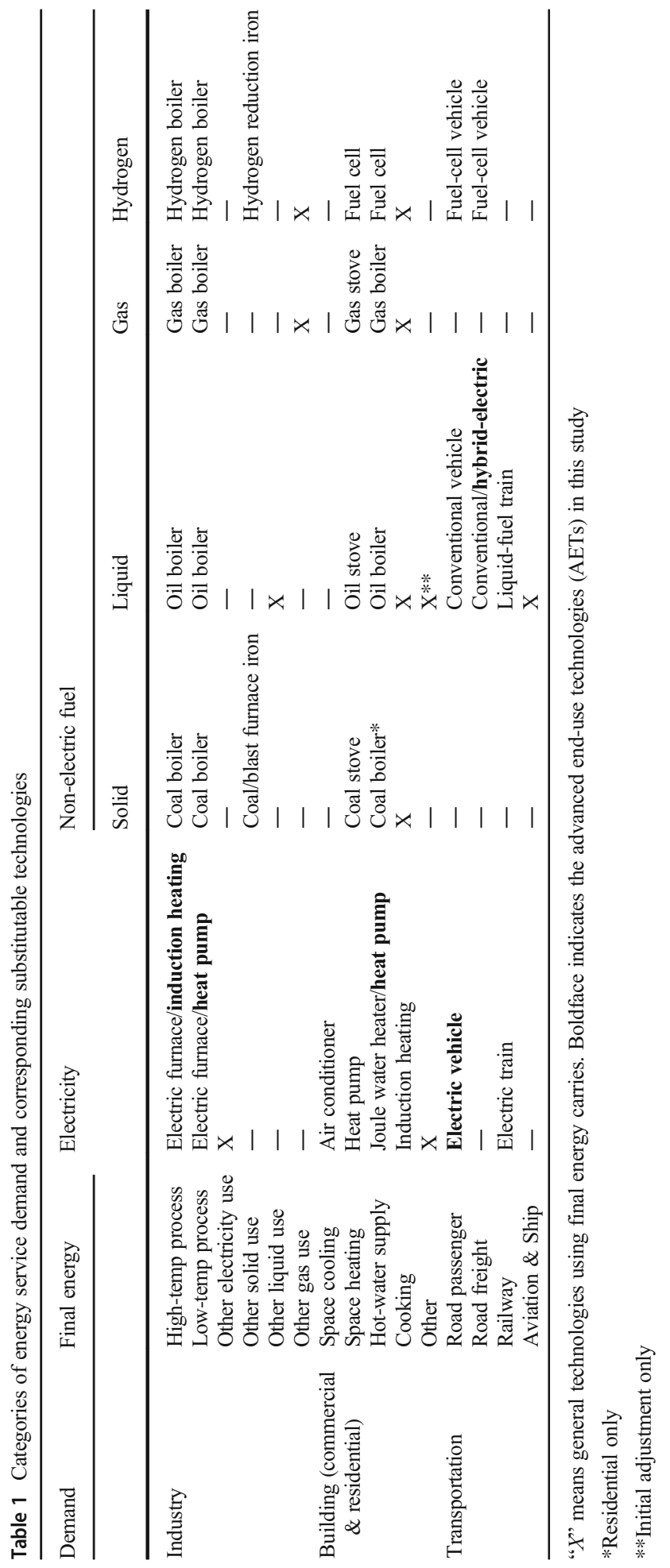


$100 \%$ bio-electricity by conventional power generation, (2) biogas by high-temperature gasification or methane digestion, (3) liquid biofuel by fermentation, and (4) solid biomass. Currently, a CCS option is available only for the $100 \%$ bio-electricity. Tables in the ESM contain the related conversion costs and efficiencies.

The model system is solved as a nonlinear optimization maximizing the weighted sum of the regional discounted utilities. The weighting factors, termed Negishi weights (Negishi 1960; Leimbach and Toth 2003), are iteratively determined so that the discounted quantities of the regional trade balance converge to zero. The discount rate, which is defined as the rate of social time preference, is set to $3 \%$ per year at the beginning and is slightly decreased by a factor of 0.95 every 30 years. Besides, capital costs are discounted with a real interest rate, which is regionally set to the sum of the social discount rate and the growth rate of reference GDP.

According to the EMF-33 scenario protocols (ESM section 4), the mitigation policy is globally implemented for $\mathrm{CO}_{2}$ emissions from fossil fuels and industry (FFI) by either pricing or budget. As described in the next section, we have conducted both types of scenario experiments for intensive analysis along the protocol. In the former price scenarios, carbon taxes with revenue recycling are imposed; in the latter budget scenarios, budget constraints are directly imposed. Although there are procedural differences between price and budget scenarios, duality is ensured so that the results become almost identical to each other when the exogenous carbon taxes in the former are equivalent to the endogenous carbon prices in the latter.

It is assumed that the amount of $\mathrm{CO}_{2}$ emissions from industrial processes, mostly from cement production (not explicitly modeled), is proportional to the sum of energy service demand in the industry and transportation sectors with a constant scaling parameter adjusted to the 2010 world total emissions.

Regarding $\mathrm{CO}_{2}$ emissions in the land-use sector, harvesting standing woods for the fuelwood feedstock is a major source in the current model configuration. To constrain land $\mathrm{CO}_{2}$ emissions, additional taxes are imposed on the use of fuelwood feedstock. This tax is calculated using a constant emission factor that is empirically determined based on the cumulative relationship of woody biomass consumption to associated emissions (ESM section 2).

Table 2 Experimental scenarios

\begin{tabular}{|c|c|c|c|}
\hline Dimension & Constraint & Variation & Label component \\
\hline Policy (P) & $\begin{array}{l}\text { Pricing by carbon } \\
\text { taxes, or budget of } \\
2011-2100 \text { FFI } \\
\text { emissions }\end{array}$ & $\begin{array}{l}\text { Tax in the range } 1-690 \mathrm{USD} / \mathrm{tCO}_{2} \text { in } \\
2030 \text { followed by a common devel- } \\
\text { opment pathway, three budgets of } \\
1600,1000 \text {, and } 400 \mathrm{GtCO}_{2} \text {, or no } \\
\text { constraints as baseline }\end{array}$ & $\begin{array}{l}\text { No label for pricing; "BHi," } \\
\text { "BLo," "BVlo" for the } \\
\text { budgets; "Base" for the } \\
\text { baseline }\end{array}$ \\
\hline $\mathrm{ABT}(\mathrm{B})$ & $\begin{array}{l}\text { Bioenergy supply limit, } \\
\text { or BECCS } \\
\text { availability }\end{array}$ & $\begin{array}{l}\text { Two limits of } 100,200 \mathrm{EJ} / \text { year, no } \\
\text { BECCS, or no constraints as full ABT } \\
\text { technologies }\end{array}$ & $\begin{array}{l}\text { "Lim1," "Lim2," "None," or } \\
\text { "Full" }\end{array}$ \\
\hline $\operatorname{AET}(\mathrm{E})$ & $\begin{array}{l}\text { Availability of } \\
\text { advanced end-use } \\
\text { technologies }\end{array}$ & Yes/No & “On”/“Off” \\
\hline
\end{tabular}

The three dimensions are identified by the letters "P," "B," and "E" in the composite labels in figure legends. The policy constraint by carbon taxes has many variations, and they are represented by coordinate axes instead of labeling in the figures 


\section{Experimental design}

To analyze in detail the role of end-use technologies on multiple dimensions, we have added a number of scenarios as an extension to the EMF-33 demand phase. Table 2 categorizes all the scenarios used in the present study by policy, ABT, and AET constraints.

The policy dimension includes the three standard budgets of the EMF-33 scenarios, an extended series of carbon taxes, and the baseline as no climate mitigation policy. The standard budgets are defined as 1600,1000 , and $400 \mathrm{GtCO}_{2}$ of 2011-2100 FFI emissions, referred to as high, low, and very low budgets, respectively; the latter two are supposed to be consistent with the 2 and $1.5^{\circ} \mathrm{C}$ goals (i.e., the limit of the global-mean temperature increase relative to the preindustrial level). The budget constraint is implemented so that it allows overshooting until 2090, hits a specific limit at 2100, and keeps the budget not to exceed the limit later. According to the EMF-33 protocol, the global FFI $\mathrm{CO}_{2}$ emissions in 2020 are constrained to $36.2 \mathrm{GtCO}_{2}$, representing a "weak" mitigation policy in BET.

The series of tax policies are designed to cover mitigation levels ranging from a near-baseline level to an extremely stringent one that reaches the $1.5^{\circ} \mathrm{C}$ goal when the bioenergy and end-use technologies are fully available. To ensure consistency with the EMF-33 protocol, the tax becomes effective in 2030, and the emissions in 2020 are constrained as in the budget scenarios. The initial tax in 2030 encompasses a wide range (1-690 USD/tCO 2 ), followed by a trajectory with a common shape that is consistent with the $\mathrm{CO}_{2}$ budget constraint. As illustrated in Fig. 1 and discussed in the next section, this tax trajectory is designed such that it increases at a fixed annual rate of $4.5 \%$ until 2070 and remains constant after a transition period of 30 years, in which the increase rate declines linearly to zero in 2100 . The intervals of the initial tax levels are set to be small enough to appropriately interpolate resulting scenarios at a specific budget size to examine how the carbon prices are adjusted to comply with the budget when the ABT and/or AET are varied.

Note that an exponentially increasing carbon tax has been proposed for the purpose of model diagnosis in Kriegler et al. (2015) because of its consistency with a growing economy.

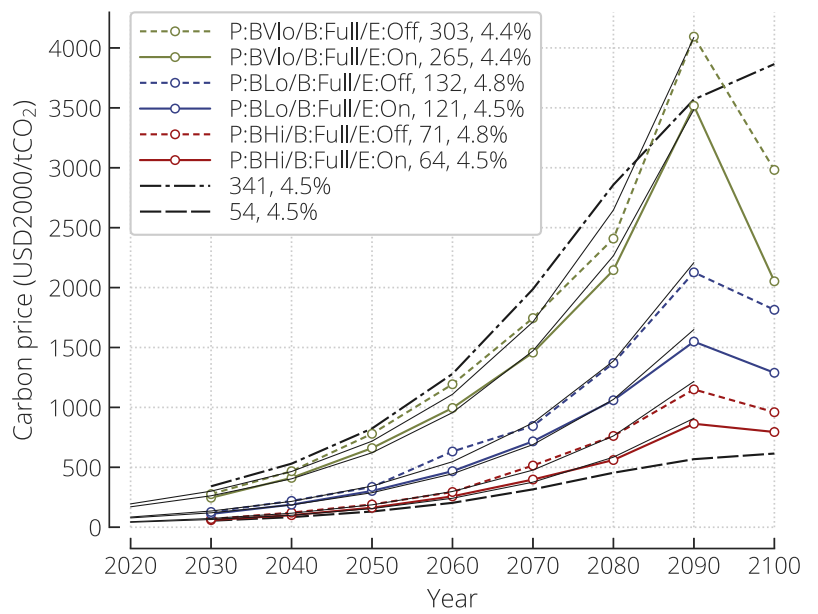

Fig. 1 Carbon prices over time for the EMF-33 budget scenarios with and without advanced end-use technologies (AETs). Thin black lines represent the exponential fitting to price changes from 2030 to 2090. (See the text for an explanation about the decline in 2100.) Black dashed and dash-dotted lines show examples of carbon taxes used in the price scenarios. Numbers in the legend are the 2030 prices in USD/ $\mathrm{tCO}_{2}$ and their annual increase rate, respectively 
On top of that, we can propose the present methodology as a proxy of a series of budget scenarios because of its simplicity in implementation.

Prices in the present study are given in year 2000 USD. This price unit is used internally, and values are adjusted so as to be consistent with the EMF-33 protocol. Because of the duality between the price and budget scenarios, an exogenous carbon tax in the former and an endogenous carbon price in the latter are interchangeably mentioned here.

The ABT dimension comprises four mutually exclusive options: no constraints (model default), primary bioenergy supply limits of 200 and $100 \mathrm{EJ} / \mathrm{year}$, and no BECCS. The bioenergy supply limit generally acts on the ABTs limitations in deep mitigation scenarios, and BECCS is effectively the only option for ABTs in the model. Thus, the constraints on the bioenergy supply and BECCS availability are both categorized into the same ABT dimension. There are two options for the AET dimension: activating (model default) and inactivating a set of high-efficiency end-use technologies, including industrial induction heaters, heat pump water heaters in industry and building sectors, hybrid trucks, and electric vehicles, as shown in Table 1. These are the same selection as in Yamamoto et al. (2014) and key substitutable technologies that cover entire end-use sectors. (See section 3 of the ESM for more details.)

In the following section, we ensure consistency of carbon prices between different climate policies and compare the resulting scenarios across different ABT/AET constraints over policy variations by continuous pricing or specific budgets, where policy and technologies are the primary and secondary dimensions, respectively. In order to refer to a specific set of scenarios, labels identifying the type of constraint for each dimension are used, as shown in Table 2. Moreover, no constraints on both ABT and AET are referred to as full technologies, while the bioenergy supply limits of 200 and $100 \mathrm{EJ} /$ year are named weakly and strongly limited supply, respectively.

\section{Results and discussions}

\subsection{Budget constraints and resulting carbon prices}

Figure 1 shows the changes in the endogenous carbon prices over time in the scenarios constrained by the high, low, and very low budgets (labeled P:BHi, P:BLo, and P:BVlo, respectively), with and without the AETs (labeled E:On and E:Off, respectively), where the ABT is not constrained (labeled B:Full). The price in each scenario increases exponentially before declining in 2100 . The annual growth rate is approximately constant at about $4.5 \%$ regardless of the budget size. Although the lack of the AETs causes a rise in the growth rate, except for the very low budget case, the difference is within a few tenths of percent point.

The exponential increase toward the end of the century results from inter-temporal optimization under the budget constraint and can be explained by analogy with the Hotelling rule on exhaustible energy resources. The growth rate of $4.5 \%$ is close to the long-term interest rate defined as the sum of the social discount rate and the growth rate of reference GDP (Fig. ESM.3). The carbon prices discontinuously drop in 2100 , which also results from the optimization where the budget constraint is imposed in 2100 and thereafter. Similar discontinuous drops were found in a stabilization scenario in terms of the atmospheric $\mathrm{CO}_{2}$ concentration (Weyant 2004). One implication is that a stringent budget constraint at an assumed target year greatly affects the budget overshooting, as discussed in Obersteiner et al. (2018). Although this is not within the scope of the present study, it raises an issue of allowable temperature overshooting with regard to the mitigation policy. 


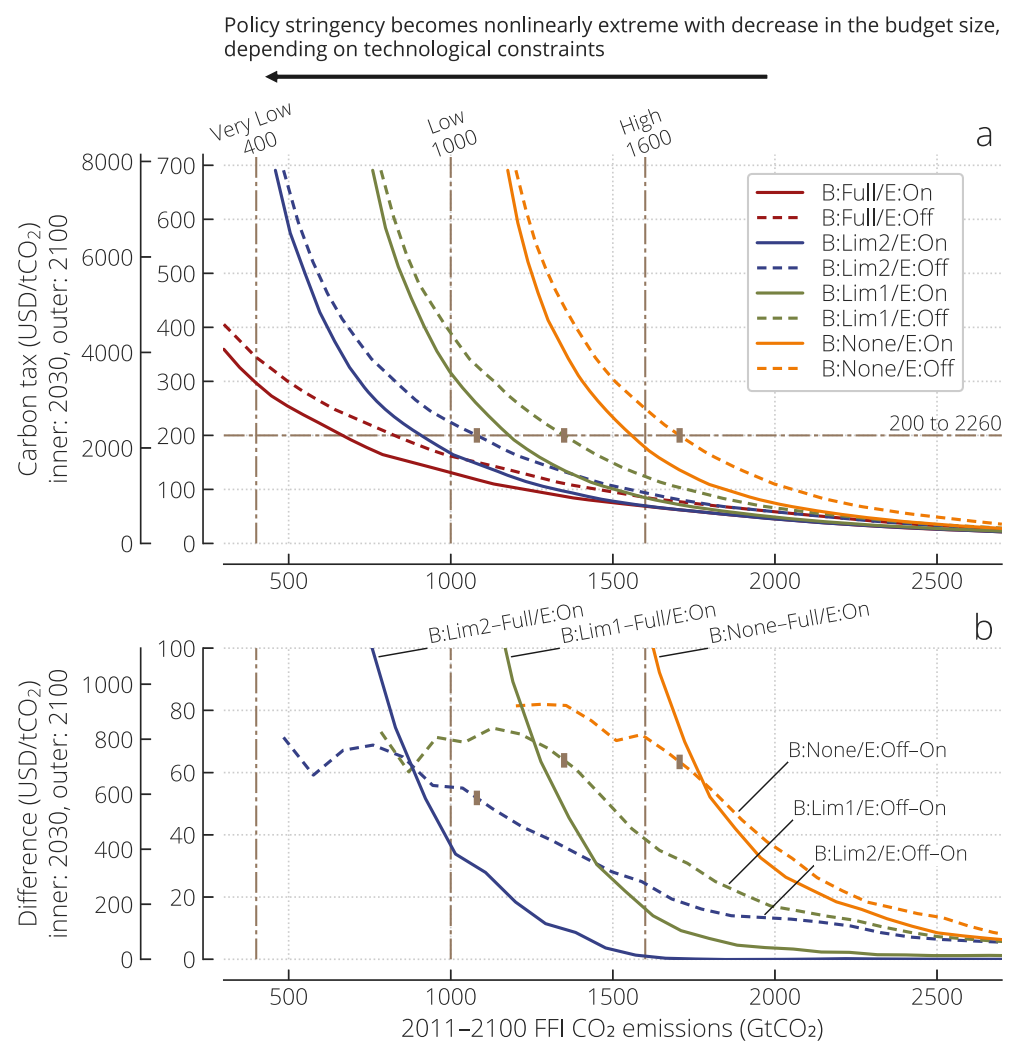

Fig. 2 Relationship between policy cost by carbon taxes in 2030 and 2100 (vertical axes) and $\mathrm{CO}_{2}$ budgets by 2011-2100 cumulative fossil fuels and industry (FFI) emissions (horizontal axis). In panel a, sets of scenario series with and without the advanced end-use technologies (AETs) are shown by solid and dotted lines, respectively, for different advanced bioenergy technologies (ABTs) constraints represented by colors. In panel b, inter-series differences in terms of the ABT constraints are examined for the series with the AETs relative to the full-technology series (red solid line in panel a) and shown by solid lines. These are compared with interseries differences in terms of the AET constraints for each ABT-constrained series shown by dotted lines. Imposed taxes start from 2030 and increase by about a factor of 11.3 in 2100 . The vertical dash-dotted lines correspond to the high, low, and very low budgets. The horizontal dash-dotted line in panel a indicates a tax level of $200 \mathrm{USD} / \mathrm{tCO}_{2}$ in 2030 for diagnostic use, and the rectangle markers guide the AET sensitivity for each ABT constraint at the budget corresponding to the specific tax level

\subsection{Relationship between policy stringency and mitigation levels}

Figure 2 shows the relationship between the policy stringency, measured by carbon taxes in 2030 and 2100, and the resulting degree of climate mitigation, measured by the $\mathrm{CO}_{2}$ budget of 2011-2100 FFI emissions, for each scenario series and differences between paired series across the ABT and the AET dimensions. The budget is shown over the range of 300-2700 $\mathrm{GtCO}_{2}$. The ratio of the tax in 2100 to that in 2030 is about 11.3 in the common tax trajectory.

As shown in Fig. 2a, the price-budget relationship is generally nonlinear, in such a way that the reduction of the budget by an additional tax increment diminishes as the tax level increases. In the full-technology series (B:Full/E:On), an increase in the tax needed to save $600 \mathrm{GtCO}_{2}$ approximately doubles from the high-to-low budget to the low-to-very low budget. A similar 
nonlinear tendency has been found in the diagnostic study by Kriegler et al. (2015). They have derived a convex-shaped marginal abatement cost curve from only two data points with different initial carbon taxes. The multiple data points in the present study build smooth curves, which enable a more detailed quantitative analysis. Since there is a one-to-one correspondence between the imposed tax scaling and the resulting cumulative emissions for a given set of technology constraints, we can discuss the results in the following in terms of $\mathrm{CO}_{2}$ budgets for 2011-2100 cumulative emissions and carbon prices referred to as the tax values in 2030.

Although the budget size can be reduced to those around the very low level $\left(400 \mathrm{GtCO}_{2}\right)$ within the range of tax scaling considered here, the policy stringency becomes extreme in such deep mitigation. When the ABT constraint is imposed and becomes severe, the price increase becomes steeper, implying that severe $\mathrm{ABT}$ constraints render low $\mathrm{CO}_{2}$ budgets unattainable from technological and economic concerns. This result is viewed as a horizontal shift of the pricebudget curve, as found in Azar et al. (2013) and Luderer et al. (2013). The series without the AET (E:Off) basically follow the corresponding series with the AET (E:On) but are altered so that the price consistently increases. Different from the ABT impact, the AET impact is viewed as a vertical shift of the curve. Thus, the ABT has implications for attainable low carbon scenarios while the AET makes policy instruments less stringent without regard to the ABT constraints.

No BECCS series (B:None) can attain the high budget level, but not the low level. Since electricity with BECCS is the only option to realize negative emissions in BET, the quantity of available bioenergy that can be allocated to BECCS, or BECCS potential, primarily determines the extent of the budget reduction. Note, however, that whether the model finds a computational solution without negative emissions depends on how to implement the policy constraints. In fact, BET fails to produce solutions without BECCS when the high budget constraint is directly imposed (Table 2 in Bauer et al. 2018), instead of indirectly by pricing, due to the difficulties in seeking solutions under net-zero emissions throughout the model period beyond 2100. Although this difference is a model feasibility matter, it has an indication of technological and economic challenges.

Here, to discuss different emission results for the same level of policy stringency, we focus on a tax level of $200 \mathrm{USD} / \mathrm{tCO}_{2}$ in 2030 as an economic cut-off value for the carbon tax, indicated by the horizontal dash-dotted line in Fig. 2a, for a diagnostic purpose. This tax level corresponds to $4-5 \%$ of the cumulative GDP loss, shown in the next subsection. Within this level, the budget size can be reduced to $670,910,1180$, and $1560 \mathrm{GtCO}_{2}$, respectively, in the full technology (B:Full), weakly constrained (B:Lim2), strongly constrained (B:Lim1), and no BECCS (B:None) series with the AET. In the corresponding series without the AET, it can be reduced to $830,1080,1350$, and $1710 \mathrm{GtCO}_{2}$. Namely, the range of the attainable budgets across the ABT dimension with the AET $-890 \mathrm{GtCO}_{2}$ - is comparable with that without the AET $-880 \mathrm{GtCO}_{2}$. Moreover, the differences between the attainable budgets with and without the AET -150 to $170 \mathrm{GtCO}_{2}$ - are also comparable across the ABT dimension. These results indicate that the effect of the AET on the budget size does not much depend on the ABT constraints.

Figure $2 \mathrm{~b}$ illustrates increases in carbon price due to the lack of the AET and due to the ABT constraints. These additional prices, quantifying sensitivities to each of AET and ABT, indicate that the AET sensitivity is greater than the ABT sensitivity as far as the steepness of the price-budget curve is moderate. At the budget sizes corresponding to the tax level of 200 $\mathrm{USD} / \mathrm{tCO}_{2}$ in 2030, the AET sensitivity, indicated by the markers in Fig. 2b, is still dominant over the ABT sensitivity in the weakly and strongly constrained ABT series, or comparable 
with that in the no BECCS series. Increased tax levels are 52, 64, and $63 \mathrm{USD} / \mathrm{tCO}_{2}$ at those specific budget sizes in the weakly constrained, strongly constrained, and no BECCS series, respectively. These numbers correspond to about $30 \%$ savings in the carbon price from the use of the AETs.

Note that the numbers described here actually depend on the selected AETs and the corresponding efficiency assumptions, and therefore the findings from our scenario exercise entail these uncertainties. It is nevertheless implied that there is room for compensating bioenergy restrictions to a considerable extent by further improving end-use efficiencies.

Besides the selection of AETs and their efficiency estimates, demand-side solutions essentially involve different disciplines, as proposed in Creutzig et al. (2018). In this broader context, the approach of the current study, dealing with demand-side contributions and interaction with supply sectors, should involve other relevant aspects, such as transition theory and behavioral science, as well as bottom-up assessments from technological studies.

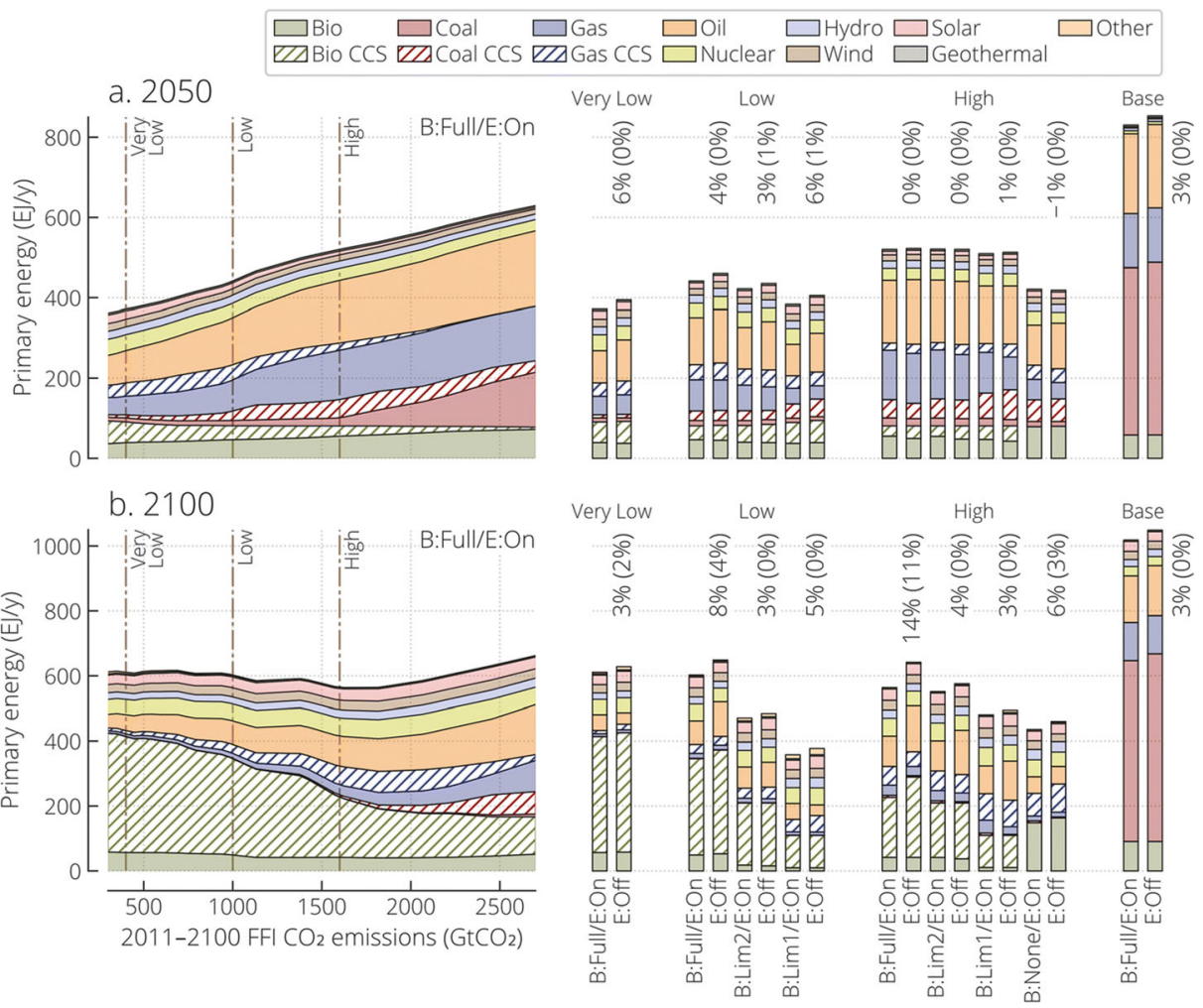

Fig. 3 Changes in primary energy supply in 2050 (a) and 2100 (b) in terms of the $\mathrm{CO}_{2}$ budget by 2011-2100 cumulative fossil fuels and industry (FFI) emissions. The left panels show the full-technology series (B:Full/ E:On) over the same range as in Fig. 2; the right panels compare multiple series at the high, low, and very low budgets as well as at the baseline, where bar charts are presented for the series that attain the specific budget levels. Attached numbers in percentage and those in parentheses represent changes by inactivating advanced enduse technologies (AET) in the total primary energy and the share of bioenergy in those changes, respectively. Biomass without CCS ("Bio" in the legend) includes exogenous traditional use, about 14 and 9 EJ/year in 2050 and 2100, respectively. Cumulative FFI $\mathrm{CO}_{2}$ emissions in the baseline are 5566 and $5699 \mathrm{GtCO}_{2}$ in the fulltechnology and its variant without the AET (B:Full/E:Off) series 
Integrated assessment modeling that incorporates these aspects would provide further insight into the role of AETs.

\subsection{Impact on energy, economy, and land-use emissions}

Figure 3 illustrates the changes in primary energy supply in 2050 and 2100 in the fulltechnology series over the same range of the $\mathrm{CO}_{2}$ budget as in Fig. 2, and the comparisons between the different series at the high, low, and very low budgets (right panels). In the middle of this century, the total primary energy decreases in response to suppressed final energy (Fig. ESM.6) as the budget decreases, while the biomass supply is almost stable at about 80-90 EJ/ year. By contrast, at the end of the century, the decrease in the total primary energy occurs up to the high budget level, and further budget reduction leads to a rapid increase in BECCS, which in turn results in a slight increase in total primary energy. Since climate change mitigation is closely connected to increase in electrification and the decarbonization of electricity including the deployment of BECCS, the total electricity generation is rather stable over the budget range while the transition in electricity generation mix are similar to those in the primary energy mix (Figs. ESM.7 and ESM.8).

To what extent such BECCS-intensive transformation occurs may differ across models. Compared with other EMF-33 models, BET tends to reallocate bioenergy to BECCS rather than to expand the total bioenergy in 2050 and to use less fossil fuels with CCS throughout this century (Bauer et al. 2018). The strong dependency on BECCS is also associated with a constraint on the share of variable renewable energy by solar and wind, which is set to $30 \%$ in terms of electricity generation in each region (Fig. ESM.7).

The lack of AETs affects the energy system in two ways: on the one hand, it causes an increase in energy supply because of more energy input to produce the same level of energy services, but on the other hand, its macroeconomic impact can bring a decrease in the level of energy services (Figs. ESM.6 and ESM.9). As demonstrated in the previous study (Yamamoto et al. 2014), this compensation basically results in a net increase in total final energy, and thereby in higher total primary energy use.

However, such an impact of AETs quantitatively differs across time and between budgets. In 2050, the increase is negligible at the high budget size, while it is 3-6\% at the other budget sizes and the baseline. (See attached numbers in percentage beside the bar charts in Fig. 3.) The AET impact in the high budget scenario becomes significant in 2100; in particular, total primary energy increases by more than $10 \%$ in the full-technology series and is associated with a relatively large deployment of BECCS (Fig. ESM.10). This exceptional behavior is attributable to the model's delayed response to moderate mitigation policies. The share of bioenergy in the increased total primary energy also differs across time and between budgets. (See percentage numbers in parentheses in Fig. 3.) While changes in the share are negligible in 2050, it shows relatively large increases in 2100 in the cases without the ABT constraints, at the high budget in particular, and in the case without BECCS. These tendencies imply that the AET reduces total primary energy consumption, and thereby help saving bioenergy resources depending on timing and the level of policy stringency.

The reduction of the $\mathrm{CO}_{2}$ budget and associated large-scale BECCS deployment affects the global economy and land-use change $\mathrm{CO}_{2}$ emissions. As typical examples of such impact, Fig. 4 illustrates the policy cost in terms of cumulative GDP losses and land $\mathrm{CO}_{2}$ emissions over the period 2011-2100, where multiple series are arranged on the FFI $\mathrm{CO}_{2}$ budget axis. 

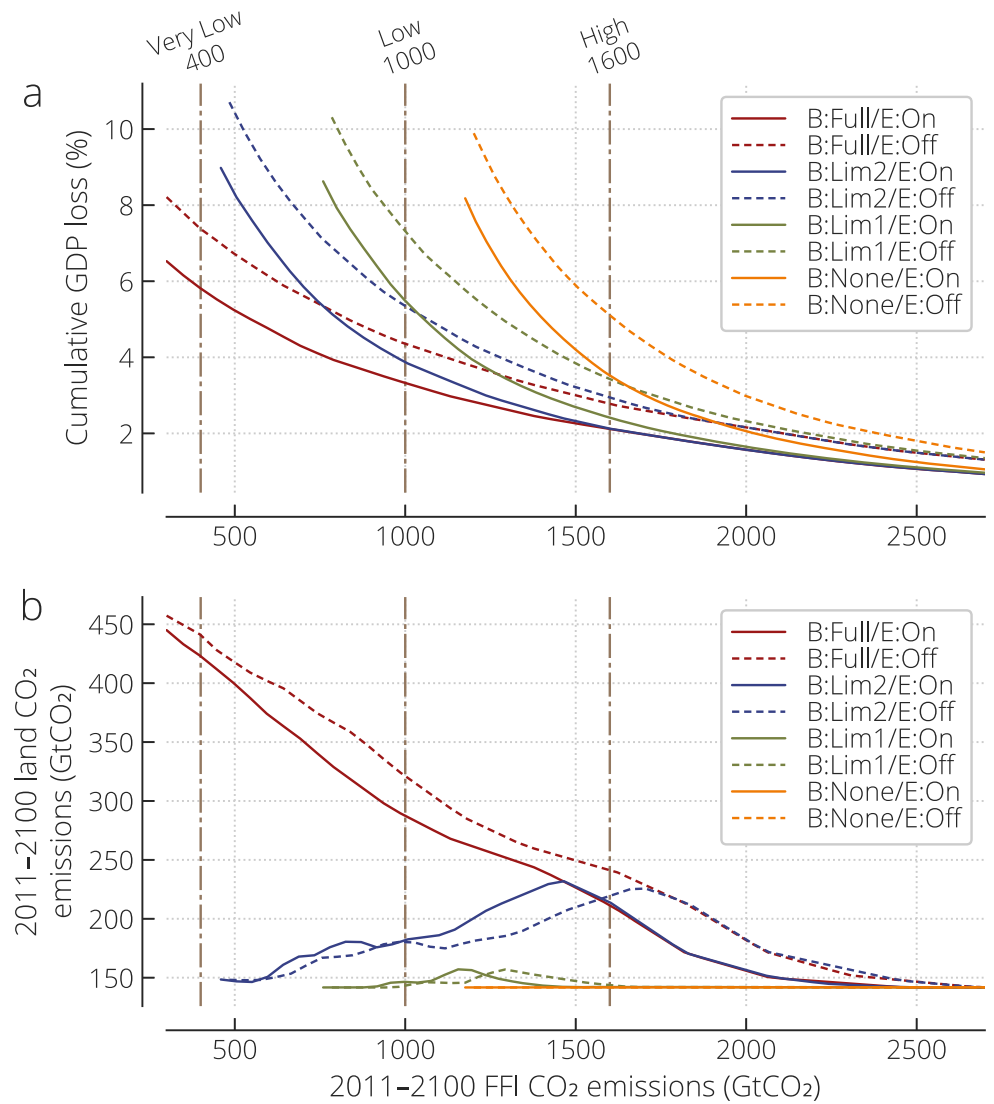

Fig. 4 Inter-series comparison of 2011-2100 cumulative GDP losses (a) and land $\mathrm{CO}_{2}$ emissions (b) on the fossil fuels and industry (FFI) $\mathrm{CO}_{2}$ budget axis with the same range as in Fig. 2. The cumulative GDP loss is defined as a sum of GDP reduction over the period relative to the full-technology baseline GDP with a discount rate of $5 \%$ per year

The cumulative GDP loss is defined as a net present value GDP reduction over the period relative to the full-technology baseline GDP applying a discount rate of 5\% per year.

The policy cost monotonically increases with the reduction of the $\mathrm{CO}_{2}$ budget, and the sensitivity to AET is significant over a wide range of budget sizes regardless of the ABT constraints. This tendency is qualitatively similar to that found in the previous study (Yamamoto et al. 2014). Basically, the sensitivity in the policy cost is consistent with that in the carbon taxes in Fig. 2a. One notes, however, that increases in the policy costs with the budget reduction in the series of $\mathrm{ABT}$ constraints are not so extreme as those in the carbon prices, which makes the AET sensitivity relatively large. These sensitivities are mostly the same as those examined over the second half of this century, except for the magnitude (Fig. ESM.11). The policy costs due to the budget reduction and their inter-series differences over the latter period are approximately twice as large as those over the whole period.

In terms of land $\mathrm{CO}_{2}$ emissions, the difference between the full and the constrained ABT series is highlighted. While changes in the land-use sector, in particular forest areas, are in line with the pricing in energy sectors, the large-scale deployment of bioenergy to reduce fossil fuel 
use induced by carbon pricing in turn leads to an increase in land $\mathrm{CO}_{2}$ emissions. As illustrated in Fig. $4 \mathrm{~b}$, in the full ABT series, the cumulative amount of land $\mathrm{CO}_{2}$ emissions monotonically increases with the reduction of the FFI $\mathrm{CO}_{2}$ budget and exceeds $400 \mathrm{GtCO}_{2}$ at the very low budget, which means that land emissions are comparable with FFI emissions. By contrast, in the weakly constrained series, it peaks at about $230 \mathrm{GtCO}_{2}$ at a level around the high budget and turns to decline beyond that although the FFI budget does not reach the very low level.

These different tendencies can be attributed to the biomass supply limit imposed in the constrained series, where the model suppresses the most $\mathrm{CO}_{2}$-intensive biomass resource (fuelwood in the case of BET) more effectively with the reduction of FFI $\mathrm{CO}_{2}$ budget (Fig. ESM.10). Without such limitation, a stringent mitigation policy may become less effective due to a larger land-use contribution to the total $\mathrm{CO}_{2}$ emissions (Fig. ESM.12). Although the model's behavior is subject to uncertainties on relevant factors of land $\mathrm{CO}_{2}$ emissions, the result implies that pricing may bring about leakage effects from the energy to the land-use sector, depending on the ABTs availability.

\section{Concluding remarks}

We have investigated the role of AETs by considering the interlinkage between primary bioenergy and energy end-use, mainly featured by BECCS and end-use electrification, respectively. Multiple series of scenarios have been created using BET-GLUE with different assumptions for the ABTs and AETs and with many different levels of policy stringency by pricing as well as by carbon budgets. The series of price scenarios have been designed to be consistent with budget scenarios that impose constraints on cumulative $\mathrm{CO}_{2}$ emissions from FFI at the end of this century.

Policy stringency for climate mitigation is nonlinearly related to the $\mathrm{CO}_{2}$ budget so that the reduction of the budget by an additional increase in carbon prices diminishes as the level of carbon prices increases. Although this finding is not a novelty, multiple series of nonlinear price-budget curves built on many data points provide a basis for a quantitative comparison of the model's sensitivities to the ABT and AET assumptions. The model results highlight contrasting differences between the ABT and AET sensitivities: the carbon price reaches an extreme value at larger budgets with the ABT constraints while it consistently increases with the lack of the AETs. Therefore, the ABT is most responsible for reducing the budget and has implications for technological and economic challenges of low carbon-budget scenarios. Although the role of the AET is secondary in reducing the budget, its effect on the carbon price does not much depend on the budget size and is dominant over the ABT's effect as far as the nonlinearity of the price-budget relationship is moderate. Overall, the AET has a significant effect on the required level of policy stringency for a given climate goal, so that it can compensate for the biomass constraints.

In response to pricing for climate mitigation, the model generally tends to use more bioenergy and allocate it to BECCS toward the end of this century. In this respect, constraints on the ABTs affect the amount of bioenergy and its BECCS allocation, and the AETs reduce the total primary energy, thereby saving bioenergy. These three factors-policy, ABTs, and AETs - interact in the process of inter-temporal optimization, resulting in some distinct impact on energy system and land-use sector despite similarities between policy stringency and resulting policy costs. The role of the AET in reducing the total primary energy quantitatively differs across time and between budgets, which basically reflects the model's overshooting 
nature depending on the ABT constraints. Regarding the total $\mathrm{CO}_{2}$ emissions, pricing may bring about leakage effects from the energy to the land-use sector, which also depends on the ABT constraints.

These findings would be made clearer by sensitivity experiments on selected AETs and assumed efficiencies as well as on the variation of final energy intensity. The share of variable renewables, which affects the degree of ABT dependence and is limited to $30 \%$ in this study, should be explored considering electricity storage technologies and demand-side flexibility. Comparing multiple IAMs with the methodology presented in this study would lead to more robust findings. Although the budget constraints involve some unnatural aspects, carefully designed price constraints for each IAM can provide alternative scenarios that are more suitable for model comparisons. Other issues emerging in the present study, such as allowable overshooting and dependency on the assumed target year, are worth being addressed for a deeper understanding.

Acknowledgments We acknowledge the steering committee and the members of EMF-33, in particular Nico Bauer, for leading the work in the demand phase of the study and for the constructive comments on this paper. We also thank Yu Nagai for helpful comments and suggestions.

Funding information This work was partially supported by the Program for Risk Information on Climate Change (SOUSEI) and the Integrated Research Program for Advancing Climate Models (TOUGOU) Grant Number JPMXD0717935715 from the Ministry of Education, Culture, Sports, Science and Technology (MEXT), Japan.

Open Access This article is licensed under a Creative Commons Attribution 4.0 International License, which permits use, sharing, adaptation, distribution and reproduction in any medium or format, as long as you give appropriate credit to the original author(s) and the source, provide a link to the Creative Commons licence, and indicate if changes were made. The images or other third party material in this article are included in the article's Creative Commons licence, unless indicated otherwise in a credit line to the material. If material is not included in the article's Creative Commons licence and your intended use is not permitted by statutory regulation or exceeds the permitted use, you will need to obtain permission directly from the copyright holder. To view a copy of this licence, visit http://creativecommons.org/licenses/by/4.0/.

\section{References}

Azar C, Johansson DJA, Mattsson N (2013) Meeting global temperature targets - the role of bioenergy with carbon capture and storage. Environ Res Lett 8:034004

Bauer N et al (2018) Global energy sector emission reductions and bioenergy use: overview of the bioenergy demand phase of the EMF-33 model comparison. Clim Chang. https://doi.org/10.1007/s10584-018-2226-y

Clarke L et al. (2014) Assessing transformation pathways. In: Edenhofer O et al. (eds) Climate change 2014: mitigation of climate change. Contribution of Working Group III to the Fifth Assessment Report of the Intergovernmental Panel on Climate Change. Cambridge University Press, Cambridge, United Kingdom and New York, NY, USA, pp. 413-510

Creutzig F, Roy J, Lamb WF et al (2018) Towards demand-side solutions for mitigating climate change. Nat Clim Chang 8:268-271

Edelenbosch OY et al (2017) Decomposing passenger transport futures: comparing results of global integrated assessment models. Transp Res Part D: Transp Environ 55:281-293

Edmonds J et al (2013) Can radiative forcing be limited to $2.6 \mathrm{Wm}^{-2}$ without negative emissions from bioenergy AND $\mathrm{CO}_{2}$ capture and storage? Clim Chang 118:29-43

Grubler A et al (2018) A low energy demand scenario for meeting the $1.5^{\circ} \mathrm{C}$ target and sustainable development goals without negative emission technologies. Nat Energy 3:515-527 
Güneralp B et al (2017) Global scenarios of urban density and its impacts on building energy use through 2050. Proc Natl Acad Sci 114:8945-8950

IEA (2017) Energy technology perspectives 2017: catalysing energy technology transformations. International Energy Agency

IEA (2018) World energy outlook 2018. International Energy Agency

Kermeli K, Graus WHJ, Worrell E (2014) Energy efficiency improvement potentials and a low energy demand scenario for the global industrial sector. Energy Efficiency 7:987-1011

Krey V, Luderer G, Clarke L, Kriegler E (2014) Getting from here to there - energy technology transformation pathways in the EMF27 scenarios. Clim Chang 123:369-382

Kriegler E et al (2014) The role of technology for achieving climate policy objectives: overview of the EMF 27 study on global technology and climate policy strategies. Clim Chang 123:353-367

Kriegler E et al (2015) Diagnostic indicators for integrated assessment models of climate policy. Technol Forecast Soc Chang 90:45-61

Leimbach M, Toth FL (2003) Economic development and emission control over the long term: the ICLIPS aggregated economic model. Clim Chang 56:139-165

Loulou R, Goldstein G, Noble K (2004) Documentation for the MARKAL family of models. Energy Technology Systems Analysis Programme. http://www.etsap.org/tools.htm

Luderer G, Pietzcker RC, Bertram C et al (2013) Economic mitigation challenges: how further delay closes the door for achieving climate targets. Environ Res Lett 8:034033

Mittal S, Dai H, Fujimori S, Hanaoka T, Zhang R (2017) Key factors influencing the global passenger transport dynamics using the AIM/transport model. Transp Res Part D: Transp Environ 55:373-388

Negishi T (1960) Welfare economics and existence of an equilibrium for a competitive economy. Metroeconomica 12:92-97

Obersteiner M, Bednar J, Wagner F et al (2018) How to spend a dwindling greenhouse gas budget. Nat Clim Chang 8:7-10

Smith P et al (2016) Biophysical and economic limits to negative $\mathrm{CO}_{2}$ emissions. Nat Clim Chang 6:42-50

Sugiyama M, Akashi O, Wada K, Kanudia A, Li J, Weyant J (2014) Energy efficiency potentials for global climate change mitigation. Clim Chang 123:397-411

van Vuuren DP, Deetman S, van Vliet J, van den Berg M, van Ruijven BJ, Koelbl B (2013) The role of negative $\mathrm{CO}_{2}$ emissions for reaching $2{ }^{\circ} \mathrm{C}$-insights from integrated assessment modelling. Clim Chang 118:15-27

Weyant JP (2004) Introduction and overview. Energy Econ 26:501-515

Yamamoto H, Fujino J, Yamaji K (2001) Evaluation of bioenergy potential with a multi-regional global-landuse-and-energy model. Biomass Bioenergy 21:185-203

Yamamoto H, Sugiyama M, Tsutsui J (2014) Role of end-use technologies in long-term GHG reduction scenarios developed with the BET model. Clim Chang 123:583-596

Publisher's note Springer Nature remains neutral with regard to jurisdictional claims in published maps and institutional affiliations. 